\title{
INCUBADORA DE EMPRESAS DE BASE TECNOLÓGICA: PERCEPÇÃO DOS EMPRESÁRIOS QUANTO AOS APOIOS RECEBIDOS
}

\author{
Loyce Graycielle de França Barbosa \\ Graduação em Administração de Empresas pela Universidade de Brasília - UNB \\ loycebarbosa@gmail.com (Brasil)
}

\section{Valmir Emil Hoffmann}

Doutorado em Administração de empresas pela Universidad de Zaragoza, Espanha

Professor Titular da Universidade de Brasília - UNB

ehoffmann@unb.br(Brasil)

\section{RESUMO}

Este estudo avalia as formas de apoio como atrativo para o ingresso no processo de incubação e para a consolidação das empresas. Em empresas inovadoras as falhas gerenciais como precificação, inserção mercadológica e pouco conhecimento de mercado e os custos iniciais relacionados a sua instalação contribuem para sua mortalidade. As incubadoras de empresa objetivam reduzir essa mortalidade por meio da capacitação gerencial, infraestrutura física e consultorias. As incubadoras de base tecnológica reúnem empresas cujos produtos e processos são gerados a partir do resultado de pesquisa aplicada na qual a tecnologia representa um alto valor agregado. Foi realizado um este estudo de caso na Incubadora de Base Tecnológica da Universidade de Brasília. Os dados foram coletados por meio da metodologia The World Cafe e por questionários aplicados via internet. Os dados foram tratados com análise de conteúdo e estatística descritiva. O critério de escolha do participante foi ser gestor de empresa incubada ou graduada. Os participantes avaliaram os serviços prestados pela incubadora e seu impacto como atrativo e para consolidação da empresa e as repostas foram correlacionadas com o faturamento das empresas. Os resultados apontaram diferenças entre as opiniões de empresas com maior e menor faturamento. Como conclusão, o caso aponta que os apoios mudam o grau de importância, do momento que são atrativos, para aquele que podem auxiliar a consolidação do negócio, variando também de acordo com o faturamento das empresas participantes.

Palavras chave: Incubadora de empresas; Empresas de Base tecnológica; Inovação. 



\section{INTRODUÇÃO}

O movimento mundial de incubadoras teve início em 1959 no Batavia Industrial Center em Nova York que foi popularmente chamado de primeira incubadora de empresas dos Estados Unidos. Entretanto o número de incubadoras não sofreu grande expansão, pelos menos até o final dos anos de 1970 (NBIA, 2009). No início dos anos 1980, 12 incubadoras estavam em operação nos Estados Unidos (NBIA, 2009).

Nas décadas seguintes, outros países ingressaram no movimento de incubadoras de empresas. Na China, bem como no Brasil, o movimento de incubadoras de empresas ganhou força no final de década de 1980 (Hackett \& Dilts, 2004); ao passo que na Colômbia a instituição mais tradicional foi fundada em 1994 (Bulla \& Gartner, 2004); período no qual as incubadoras também começaram a surgir no México (Hernández \& Estrada, 2006).

A discussão que trata do processo de inovação em pequenas e médias empresas (PME) envolve ao menos dois aspectos. O primeiro se refere à mortalidade desse tipo de empresas e o segundo ao apoio que pode ser fornecido para a inovação. Segundo o Serviço Brasileiro de Apoio às Micro e Pequenas Empresas (SEBRAE), o alto índice de mortalidade dessas empresas é causado, em grande parte, pelas falhas gerenciais (SEBRAE, 2004). Em se tratando de produtos e/ou processos inovadores, fatores como dificuldade em mapear mercado e perfil do cliente, precificação, questões de propriedade intelectual e falta de informação sobre captação de recursos somam-se à necessidade de treinamento gerencial.

Sobre o apoio à inovação nas PMEs, as incubadoras de empresas surgem como mecanismo que tem "o objetivo de melhorar o ambiente competitivo das empresas" (Vedovello \& Figueiredo, 2005, p.3) e de garantir maior sobrevida para empresas inovadoras, por meio de capacitação gerencial dos empresários incubados. Elas também foram definidas pelo Programa Nacional de Apoio as Incubadoras de Empresas, do Ministério da Ciência, Tecnologia e Inovação do Governo Federal (MCTI, 2000, p.6) como um " [...] um mecanismo que estimula a criação e o desenvolvimento de micro e pequenas empresas industriais ou de prestação de serviços, de base tecnológica ou de manufaturas leves por meio da formação complementar do empreendedor em seus aspectos técnicos e gerenciais [...]”.As incubadoras constituem um ambiente flexível, propício ao desenvolvimento de um empreendimento nascente e facilitam o acesso ao conhecimento gerado nas universidades e a entidades de fomento (Raupp \& Beuren, 2006). Então, pode-se entender que as incubadoras tanto podem auxiliar empresas já existentes como fomentar sua criação.

Revista de Administração e Inovação, São Paulo, v. 10, n.3, p.208-229, jul./set. 2013. 
As atividades desenvolvidas pelas incubadoras para assistir às empresas se dividem em infraestrutura física e administrativa, prestação de serviços especializados (Medeiros, 1998) e serviços customizados para demandas específicas. Entre esses serviços especializados e/ou demandas específicas, parecem estar os fundamentos do conhecimento de processos de negócios, indicados por Studdard (2006). Isso pressupõe que o escopo de uma incubadora vai mais além da estrutura física, imóveis e equipamentos conforme Medeiros (1998). Os fatores que sustentam a incubação de empresas são: a construção e manutenção de rede de apoio eficaz, desenvolvimento de competências de equipe de comercialização de novos negócios, monitoramento e avaliação do progresso das empresas e acesso a financiamentos adequados (Patton, Warren \& Bream, 2009).

Diante disso, este estudo avalia as formas de apoio como atrativo para o ingresso no processo de incubação e para a consolidação das empresas. Este trabalho está dividido em cinco partes, esta primeira contém a introdução; na segunda está o referencial teórico; na terceira parte apresenta-se a metodologia; na quarta estão os resultados e análise e por fim, na parte final estão as conclusões, limites e recomendações.

\section{REFERENCIAL TEÓRICO}

A incubação é uma das formas de interação entre universidade e empresa. Essa relação é baseada na necessidade de transformar o conhecimento gerado na universidade em produto para a sociedade, que é mantenedora de forma direta ou indireta, de grande parte das universidades brasileiras.

A inovação tem sido apontada como responsabilidade das empresas, do governo e das universidades, o que foi chamado de hélice tríplice. Lu (2008, p. 250) define a hélice tríplice como um modelo "criado com uma tentativa de capturar e explicar a dinâmica de processos de inovação e redes complexas entre as instituições de conhecimento exploração e aproveitamento". A primeira pá da hélice é representada pelo governo enquanto prestador de serviço. Abdalla, Calvosa e Batista (2009) definem que o governo assume o papel de interventor, por meio de subsídios para ciência e tecnologia, incentivos fiscais e alfandegários, legislação e incentivos a educação e formação superior para a população.

Trevisan e Silva (2010) definem que a segunda pá é a universidade, que por sua vez cria incubadoras, novas fontes de conhecimento e é responsável estabelecimento de novas áreas de atuação. A terceira é representada pelo setor privado, que se responsabiliza pelo "desenvolvimento de produtos e de serviços inovadores, promoção da interação com os centros de transferência de tecnologia e liderança nos Revista de Administração e Inovação, São Paulo, v. 10, n.3, p.208-229, jul./set. 2013. 
processos de mudança" (Trevisan \& Silva, 2010, p.4). Trevisan e Silva (2010, p.3) destacam que "as relações entre as três hélices podem ser consideradas um componente fundamental para inovação em uma sociedade cada vez mais baseada no conhecimento".

As parcerias entre universidade e empresa têm mostrado visível crescimento. Do lado das empresas, como coloca Dagnino (2003), entre os motivos para este crescimento está o alto custo de pesquisa para o desenvolvimento de produtos e possibilidade de compartilhamento de custos e riscos. Segundo Dagnino (2003), do lado das universidades o que reforça a relação com empresas privadas são as dificuldades em conseguir recursos para a pesquisa universitária e o desejo de legitimar o trabalho acadêmico junto à sociedade.

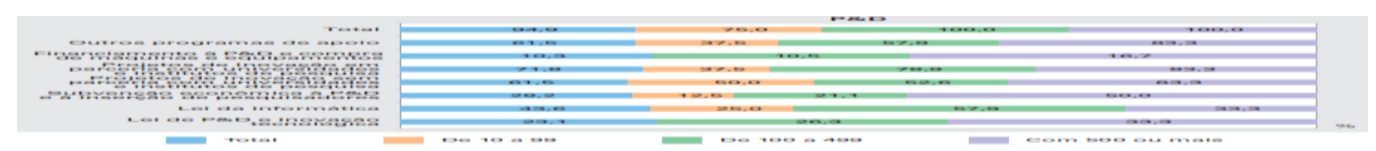

Figura1: Empresas inovadoras que utilizaram programas do governo no período de 2006-2008 por

\section{número de empregados.}

Fonte: IBGE. PINTEC - Pesquisa de inovação tecnológica: 2008. Ministério do Planejamento, Orçamento e Gestão, Instituto Brasileiro de Geografia e Estatística - IBGE, Diretoria de Pesquisas, Coordenação de Indústria. Rio de Janeiro: IBGE, 2010.

Um aumento nos investimentos públicos e privados em pesquisa e desenvolvimento é uma das estratégias que permitirão ao Brasil criar e dominar as novas tecnologias, segundo Oliva et al., (2011). O Instituto Brasileiro de Geografia Estatística, por meio da Pesquisa de Inovação Tecnológica (2008) mostrou o índice de empresas inovadoras que utilizaram programas do governo por números de empregados, e ressaltou que as médias e grandes empresas inovadoras no período (Figura 1), em sua totalidade utilizaram algum tipo de apoio do governo e que parece que se está cumprindo as recomendações de Oliva, Sobral, Santos, Almeida \& Grisi (2011).

A atuação do governo na hélice tríplice é representada também pela criação de políticas públicas que incentivem a ciência e tecnologia. No Brasil pode-se citar a Lei de Inovação (facilitação a relação universidade - empresas por meio da desburocratização na contratação de serviços) e a Lei do Bem que dá estímulos fiscais para empresas inovadoras, que visam estimular e facilitar a geração de empresas inovadoras. 


\subsection{As incubadoras em âmbito mundial e nacional}

O interesse sobre o processo de incubação tem estimulado o crescimento do número desse tipo de instituição em diversos países. No Brasil, com base em um estudo realizado pela Anprotec e pelo Ministério de Ciência e Tecnologia i Inovação (MCTI) em 2011 existem 384 incubadoras (Anprotec, 2011); na China existem aproximadamente 670 instituições em funcionamento (Tang, Angathevar \& Pancholi, 2010); nos Estados Unidos são cerca de 1000 incubadoras (NBIA, 2009); o México, conforme os estudos de Hernandéz e Estrada (2006) conta com cerca de 220 incubadoras de empresas; e na Colômbia somam 27 instituições ativas (Bulla \& Gartner, 2004). Entretanto, não é possível medir o sucesso das incubadoras apenas pela quantidade de instituições existentes. Segundo Hackett e Dilts (2004), é preciso considerar desde a equipe e gerência da incubadora, até universidades locais, rede de relacionamentos, estratégias adotadas e atuação do governo.

Para a análise em âmbito mundial é preciso considerar ainda as diferenças culturais que fazem com que os atores envolvidos desempenhem papéis diferentes em cada país. Chandra e Fealey (2009) demonstram as diferenças de estratégias sobre o processo de incubação, por meio da forma de atuação do governo no sistema de incubação dos Estados Unidos, Brasil e China (Quadro 1).

Percebem-se diferenças entre a forma de atuação do governo, fontes de financiamento e tipos de incubadoras quando se comparam os países. Em alguns aspectos, o modelo brasileiro se aproxima do estadunidense e em outros ao chinês e em outros, há grandes diferenças entre os países. Conforme Chandra e Fealey (2009), existe no Brasil mais preocupação com o fomento ao espírito empresarial (ou empreendedor) e, até pelo papel que o governo assume, com o desenvolvimento sócio-econômico e a comercialização. Em relação aos tipos de incubadora, o Brasil se aproxima mais dos Estados Unidos, onde há incubadoras mistas, de base tecnológica e especializadas. Mas se assemelha à China em relação ao papel que o governo exerce, à exceção do fato de que no Brasil o governo procura atuar de maneira mais sinérgica.

O movimento brasileiro de incubadoras teve início em 1987 com o surgimento da primeira incubadora brasileira: Secretaria de Ciência, Tecnologia e Desenvolvimento de São Paulo, seguida pelo ParqTec no estado da Paraíba e a Fundação CERTI no estado de Santa Catarina, no mesmo ano. Até o ano de 1995 somavam apenas 16 incubadoras em funcionamento (Medeiros, 1998). Segundo a Associação Nacional das Entidades Promotoras de Empreendimentos Inovadores (ANPROTEC, 2007), a

Revista de Administração e Inovação, São Paulo, v. 10, n.3, p.208-229, jul./set. 2013. 
incubadoras no Brasil estão distribuídas por 25 de 27 Unidades da Federação e mais de $40 \%$ das universidades federais contam com uma incubadora.

\begin{tabular}{|c|c|c|c|}
\hline & Brasil & China & Estados Unidos \\
\hline Foco Estratégico & $\begin{array}{c}\text { Fomento ao espírito } \\
\text { empresarial, } \\
\text { desenvolvimento } \\
\text { econômico, criação de } \\
\text { empregos e } \\
\text { comercialização. }\end{array}$ & $\begin{array}{c}\text { Missão Social, } \\
\text { desenvolvimento com } \\
\text { foco em alta tecnologia. }\end{array}$ & $\begin{array}{c}\text { Desenvolvimento } \\
\text { econômico, transferência de } \\
\text { tecnologia e } \\
\text { comercialização. }\end{array}$ \\
\hline $\begin{array}{c}\text { Patrocínio e/ou } \\
\text { Financiamento da } \\
\text { Incubadora }\end{array}$ & $\begin{array}{c}\text { Financiamento que } \\
\text { incluem diferentes níveis } \\
\text { de governo, universidades } \\
\text { e alguns fundos privados. }\end{array}$ & $\begin{array}{l}\text { O governo é a principal } \\
\text { fonte de financiamento } \\
\text { das incubadoras e de } \\
\text { empresas incubadas. }\end{array}$ & $\begin{array}{c}\text { Níveis de governo, } \\
\text { organizações voltadas para o } \\
\text { desenvolvimento econômico } \\
\text { e fundos privados. }\end{array}$ \\
\hline $\begin{array}{c}\text { Tipos de empresas } \\
\text { Incubadas }\end{array}$ & $\begin{array}{l}\text { Base tecnológica, cultural, } \\
\text { social e de design. }\end{array}$ & $\begin{array}{l}\text { Predomínio de } \\
\text { incubadoras de base } \\
\text { tecnológica. }\end{array}$ & $\begin{array}{c}\text { Mistas, de base tecnológica e } \\
\text { especializadas. }\end{array}$ \\
\hline $\begin{array}{l}\text { Serviços } \\
\text { oferecidos }\end{array}$ & $\begin{array}{c}\text { Serviços compartilhados e } \\
\text { especializados. }\end{array}$ & $\begin{array}{l}\text { Principalmente serviços } \\
\text { tangíveis e de natureza } \\
\text { administrativa. }\end{array}$ & $\begin{array}{c}\text { Serviços tangíveis e } \\
\text { especializados que geram } \\
\text { valor agregado. }\end{array}$ \\
\hline $\begin{array}{l}\text { Serviços } \\
\text { financeiros }\end{array}$ & $\begin{array}{l}\text { Linhas de financiamento } \\
\text { do governo, angel's e } \\
\text { capital de risco. } \\
\text { Investimentos diretos em } \\
\text { empresas são raros. }\end{array}$ & $\begin{array}{c}\text { Financiamentos do } \\
\text { governo, subvenção e } \\
\text { capital de risco. Na } \\
\text { região Sul, são raros os } \\
\text { casos de investimento } \\
\text { direto. }\end{array}$ & $\begin{array}{l}\text { Orientação para fontes de } \\
\text { financiamento com alguns } \\
\text { casos de investimento direto. }\end{array}$ \\
\hline Papel do governo & $\begin{array}{l}\text { Visível. Abordagem } \\
\text { sinérgica. }\end{array}$ & Alta interferência. & Baixo apoio. \\
\hline
\end{tabular}

Quadro1: Diferenças do sistema de incubação entre Brasil, China e Estados Unidos.

Fonte: Adaptado de Chandra, A. B. \& Fealey, T. (2009, December); Business incubation in United States, China and Brazil: a comparison of role of government, incubation funding and financial services. International Journal of Entrepreneurship. 
A implantação de uma incubadora deve ser justificada por um estudo de viabilidade que leve em consideração a definição do papel das instituições de apoio, perfil empresarial local, interessados no processo de incubação entre outros (Medeiros, 1998). Ademais, é preciso ter em mente que as incubadoras partem da premissa de atender a carências do empresariado local e de garantir que seu desenvolvimento ocorra de maneira planejada e estruturada (Moraes \& Firmo, 2004). Como escreve Dornelas (2002), o objetivo da incubadora é a produção de empresas de sucesso, ou seja competitivas, mesmo depois de deixarem o processo, o que geralmente ocorre entre dois e quatro anos.

Segundo a Anprotec (2007), o trabalho desenvolvido pelas incubadoras gera cerca de 30 mil postos de trabalho diretos; faturamento estimado das empresas incubadas de $\mathrm{R} \$ 400$ milhões e somatório do faturamento estimado de empresas graduadas (aquelas que concluíram com sucesso o processo de incubação) estimado em $\mathrm{R} \$ 1,6$ bilhão. Apesar de os dados não serem atualizados, os resultados positivos são reforçados pelo MCTI (2001), que constatou que a taxa de mortalidade entre as empresas que passaram pelo processo de incubação é de menos que $20 \%$, enquanto a média das empresas que não foram incubadas é de até 49\% (SEBRAE, 2004), no mesmo período.

Em seus estudos, Raupp e Beuren (2006) descrevem que no Brasil, os tipos de incubadoras mais comuns são as de base tecnológica, tradicionais e mistas, essas últimas vinculadas à demanda de cada região (cultural, setorial, social entre outros). O MCTI (2000) descreve os três tipos predominantes da seguinte maneira:

a) Incubadoras tradicionais: abrigam empreendimentos que atuam em áreas da economia cuja tecnologia encontra-se amplamente disseminada.

b) Incubadora de base tecnológica: abrigam empreendimentos cujos produtos são resultado de pesquisa aplicada. Como estas são o objeto deste estudo, seguirá uma discussão sobre suas particularidades.

c) Incubadoras mistas: abriga os dois tipos anteriores.

\subsection{Incubadoras de Base Tecnológica}

Wolffenbüttel (2001, p.23) escreve que a incubadora de base tecnológica "abriga empresas cujos produtos, processos ou serviços são gerados a partir do resultado de pesquisa aplicadas na qual a tecnologia representa um alto valor agregado". Em uma conceituação menos objetiva, Vedovello e Figueiredo (2005) definem que incubadoras de base tecnológica se diferenciam por voltarem sua atenção a empresas que promovem inovação. Esse conceito é complementado por Patton, Warren e Bream (2010, p.623), que definem a atividade de incubação de empresas de base tecnológica como a "que liga de forma

Revista de Administração e Inovação, São Paulo, v. 10, n.3, p.208-229, jul./set. 2013. 
eficaz tecnologia, capital e know how (saber fazer, ter conhecimento prático, conforme Anprotec, 2007) para alavancar o talento empreendedor, acelerar o desenvolvimento de novas empresas e, assim, a comercialização da tecnologia". Oliva, Sobral, Santos, Almeida \& Grisi (2011) definem que as incubadoras de base tecnológica têm a finalidade de aceitar e incubar novas empresas cujos processo de produção requerem o uso inovador de tecnologias e de denso conhecimento científico.

Consta no Edital de Seleção da Incubadora de Base Tecnológica da Universidade de Brasília (CDT/UnB, 2010), que as empresas que participam desta modalidade de incubação atuam em áreas de biotecnologia, nanotecnologia, biomedicina, saúde, geociências, engenharias, farmacologia, tecnologia da informação e comunicação ou outras áreas que possam interessar a região em que atua incubadora.

As empresas de base tecnológica (EBT) são caracterizadas como empreendimentos comprometidos com pesquisa e desenvolvimento de produtos e/ou processos inovadores que são fruto de aplicação sistemática do conhecimento técnico. Em comparação com outras organizações, à primeira vista, diferenciam-se pela quantidade reduzida de mão de obra e pela alta capacitação técnica. Soma-se a isso que a maioria das EBTs têm origens nos spin-off (Andino, Fracasso, Silva, \& Lobler 2004, p.3) que segundo a Anprotec (2007), são aquelas oriundas de laboratório e resultado de pesquisa acadêmica ou industrial, de projetos desenvolvidos por universidades e centros de pesquisa. São, portanto, empresas que geram produtos e/ou processos inovadores.

Inovação tecnológica é conceituada como "transformação do conhecimento em produtos, processos e serviços que possam ser colocados no mercado" (MCTI, 2000, p.4). Uma empresa que gera produtos ou processo inovadores "é uma empresa que tenha implantado produtos ou processo tecnologicamente novos com substancial melhoria tecnológica durante um período de análise” (Manual de Oslo, 2001, p.55). Conceitua-se processo inovador como métodos de produção novos ou melhorados. Produto inovador é aquele "cujas características tecnológicas ou usos pretendidos diferem daquelas dos produtos produzidos anteriormente" (Manual de Oslo, 2001, p.55).

As empresas de alta tecnologia, por serem gerenciadas por pessoas com capacidade técnica elevada, em geral não estão bem informadas sobre competências necessárias para operar um negócio, como o conhecimento sobre marketing, contabilidade, recursos financeiros e humanos (Studdard, 2006). As incubadoras de base tecnológica se propõem a preencher essas lacunas de capacitação dos gestores de empreendimentos tecnológicos e ajudá-los a enfrentar as barreiras ambientais escrevem Andino, Fracasso, Silva e Lobler (2004). 
Para que as carências dos gestores de empresas incubadas sejam supridas, observadas as necessidades dos empresários, as incubadoras oferecem consultorias, assessorias, entre outras formas de apoio. A incubação inicia-se com o processo de seleção, realização do processo de incubação em si, passando pelo apoio administrativo e por último a graduação da empresa do processo de incubação (Wolffenbütel, 2001). Segundo relatório de acompanhamento da incubadora em estudo, as empresas levam entre 3 e 5 anos para se graduarem.

Salvador, Tutida e Ceretta (2009) revelam em seu estudo que a disponibilização de espaço físico é um fator que motiva a formação de projetos de base tecnológica em incubadoras de empresas. Entretanto, não somente a concessão de espaço físico constitui o apoio oferecido pela incubadora, ela deve disponibilizar:

a) Ambiente flexível e encorajador ao empreendedorismo;

b) Capacitação e assessoria técnica e gerencial;

c) Serviços compartilhados: telefone, internet, ambientes para reuniões;

d) Informações sobre mecanismos de financiamento e instituições de fomento;

e) Acesso a redes de relacionamento.

Como resultado do processo de incubação, Wolffenbüttel (2001) destaca a transferência para o setor produtivo do conhecimento gerado; a criação e desenvolvimento de novas empresas; a capacitação técnica e gerencial das empresas e a cooperação entre a universidade e a sociedade. No âmbito da universidade, essa cooperação reflete-se no incremento da qualificação de atividades de ensino e pesquisa. Para a sociedade, referem-se ao estímulo ao desenvolvimento local, pela formação de novas empresas, complementa Wolffenbüttel (2001).

Entretanto, para que o trabalho desenvolvido pela incubadora alcance o melhor resultado possível parece necessário ajustar a oferta de serviços à demanda, ou seja, é necessário saber dos clientes da incubadora - empresários incubados - quais apoios oferecidos têm maior impacto na consolidação das empresas. Estudos mostram que os maiores atrativos para as EBTs se candidatarem a um processo de incubação é a disponibilidade de espaço físico e da possibilidade de apoio financeiro (Salvador, Tutida \& Ceretta, 2009).

\section{METODOLOGIA DA PESQUISA}

A pesquisa é descritiva por expor características da população e por não ter compromisso em explicar os fenômenos que descreve (Roesch, 2006). A pesquisa é qualificada como de campo, pois foi Revista de Administração e Inovação, São Paulo, v. 10, n.3, p.208-229, jul./set. 2013. 
realizada no local onde ocorrem os fenômenos ou que possui elementos para explicá-los (Roesch, 2006). A natureza da pesquisa é qualitativa, pois permite maior compreensão do fenômeno a ser estudado. A pesquisa também é um estudo de caso, pois examina um fenômeno contemporâneo dentro de seu contexto (Vergara, 2006).

Este estudo foi realizado no Centro de Apoio ao Desenvolvimento Tecnológico da Universidade de Brasília (CDT/UnB) que abriga o programa Multincubadora de Empresas. Além da Incubadora de Base Tecnológica, objeto deste estudo, o programa Multincubadora oferece à comunidade as modalidades de incubação tradicional, dDesign, social e solidária e arte e cultura. A escolha pelo caso se deu em função do acesso e a importância para o Distrito Federal.

A coleta de dados foi feita em três momentos. No primeiro, foi realizada uma análise de relatórios de serviços prestados pela incubadora nos últimos 3 anos. Essa análise foi necessária para a descrição dos suportes oferecidos pela incubadora. No segundo momento, foi realizada a dinâmica de aplicação da metodologia The World Café, da qual participaram gestores de empresas incubadas. O The World Café é uma estratégia inovadora de diálogos colaborativos que facilita a discussão sobre temas relevantes (Brown \& Isaacs, 2007). A dinâmica do The World Café ocorre da seguinte maneira: os participantes se distribuem entre mesas, com até cinco membros, e cada mesa elege o Anfitrião. Inicia-se então a primeira rodada. Ao encerrar a rodada, o Anfitrião permanece na mesa e os outros se dirigem a outras mesas. O Anfitrião então sintetiza as ideias discutidas para os novos membros da mesa e dá início à exposição de novas discussões. Na terceira rodada, os participantes voltam para a mesa de origem. O Anfitrião expõe o que foi discutido nas rodadas anteriores e após um momento de reflexão do grupo, é aberto um espaço de nova discussão. Dessa discussão são anotadas as principais ideias levantadas. Foram essas ideias que compuseram os diálogos anotados neste trabalho. Desse momento participaram doze das treze empresas incubadas à época da pesquisa (novembro/dezembro 2010). Note-se que não é possível identificar quem emitiu cada fala, pois se tatá de um processo coletivo.

No terceiro momento foi aplicado um instrumento construído para este estudo. O questionário foi aplicado via correio eletrônico para todas as empresas graduadas (47) e para as treze empresas incubadas Participaram representantes de empresas incubadas e também representantes de empresas graduadas (empresas que concluíram com sucesso o processo de incubação). Ao todo, doze empresas responderam o questionário, sete incubadas e cinco empresas graduadas. Para avaliar os resultados foi utilizada a técnica da escala somada.

Revista de Administração e Inovação, São Paulo, v. 10, n.3, p.208-229, jul./set. 2013. 
As informações dos questionários foram tratadas da seguinte forma: cada item do questionário representava um apoio oferecido e era medido em uma escala Osgood "itemizada" em cinco pontos. Em uma extremidade constava "Pouco importante" e na outra "Muito Importante". O cálculo foi feito com a somatória da multiplicação do score pela frequência (escala somada).

\section{RESULTADOS E DISCUSSÃO}

Os resultados são apresentados em termos de serviços ofertados, expectativas dos empresários e fatores de impacto na consolidação no negócio, com o apresentado na sequência.

\subsection{Serviços prestados}

A Incubadora de Base Tecnológica do CDT/UnB oferece às empresas incubadas os apoios descritos no Quadro 2. Medeiros (1998) descreveu os serviços prestados por incubadoras de empresas como de infraestrutura física e administrativa (espaços compartilhados, copa, recepção) e serviços especializados (consultorias e assessorias). A observação da incubadora de empresas do CDT/UnB mostrou que os serviços prestados por ela atendem à descrição de Medeiros (1998). Entretanto, o apoio oferecido pelas incubadoras não deve se restringir aos benefícios tradicionais de infraestrutura física e serviços especializados, e sim se estender à disponibilização de serviços de alto valor agregado como a criação de redes de relacionamento, como escrevem Serra, Serra, Ferreira e Fiates (2010). Ao que parece, no caso estudado os serviços com maior valor agregado são os de acesso a laboratório e relacionamentos com professores o que podem constituir uma rede de relacionamento indicada por Serra, Serra, Ferreira e Fiates (2010). 


\begin{tabular}{|c|c|}
\hline Suporte oferecido & Descrição \\
\hline $\begin{array}{l}\text { Infraestrutura } \\
\text { compartilhada }\end{array}$ & $\begin{array}{l}\text { - Salas de reunião, salas de treinamento, auditórios, serviço de copa, limpeza e } \\
\text { recepção e internet. }\end{array}$ \\
\hline $\begin{array}{c}\text { Infraestrutura da } \\
\text { universidade }\end{array}$ & - Acesso a laboratórios, relacionamento com professores e departamentos \\
\hline $\begin{array}{l}\text { Desenvolvimento de } \\
\text { perfil empresarial }\end{array}$ & $\begin{array}{l}\text { - Curso de oratória, relacionamento interpessoal, educação financeira e } \\
\text { propriedade intelectual e cursos de instituições parceiras } \\
\text { - EMPRETEC: seminário que tem por objetivo desenvolver, nos participantes, } \\
\text { características de comportamentos empreendedores. Oferecido pelo SEBRAE. } \\
\text { - Coaching Adventure: metodologia de desenvolvimento humano e } \\
\text { organizacional. }\end{array}$ \\
\hline Consultorias & Consultorias nas áreas de marketing, jurídico, finanças. \\
\hline Uso da marca & - Aplicação da marca CDT/UnB em qualquer material gráfico da empresa. \\
\hline $\begin{array}{l}\text { Acesso a outros } \\
\text { programas do CDT/UnB }\end{array}$ & $\begin{array}{l}\text { - NUPITEC: apoio em questões de propriedade intelectual. } \\
\text { - Disque Tecnologia: apoio para encontrar especialistas dentro da universidade } \\
\text { - Núcleo de Projetos: orientação para escrever projetos para editais. } \\
\text { - Programa Empresa Júnior: apoio na contratação de empresas juniores. }\end{array}$ \\
\hline
\end{tabular}

Quadro2: descrição de apoios oferecidos pela incubadora de empresa de base tecnológica.

Fonte: criação própria com base em informações disponibilizadas pela incubadora.

\subsection{Expectativas dos empresários em relação à incubação de empresas}

Parte da pesquisa buscou mostrar qual/quais dos suportes oferecidos pela Incubadora pesquisada foram mais atrativos para o ingresso da empresa no processo de incubação. As falas decorrentes da coleta de dados pela dinâmica The World Café foram:

a) Infraestrutura oferecida pela incubadora:

"Poder ter uma sala na UnB dá certa visibilidade e o preço em relação à estrutura é bom (...) isso chama a atenção".

b) Acesso à estrutura da universidade:

"No meu caso, a gente já sabia que ia precisar usar laboratório, mas para quem está fora da UnB é mais complicado conseguir bons contatos (...)". Ou ainda,

"A gente ainda não precisou usar laboratórios (...)".

Revista de Administração e Inovação, São Paulo, v. 10, n.3, p.208-229, jul./set. 2013. 
c) Capacitação do perfil empresarial:

"Os cursos de capacitação não chamaram tanto a atenção porque eu achava que não precisava desse tipo de apoio. O Coaching Adventure e o Empretec, por exemplo, eu nem imaginava do que se tratava.”

d) Acesso a consultorias oferecidas durante o processo de incubação:

"A consultoria é uma forma de receber uma orientação sobre algum tema que os sócios não dominam (...) é importante para quem está começando..."

e) Permissão de uso da marca CDT/UnB em material gráfico e sítio eletrônico da empresa;

"A marca da UnB em um sítio dá certo respeito e credibilidade para a empresa (...)"

f) Acesso a outros programas do CDT/UnB:

“A Empresa X só conheceu o trabalho da maioria dos programas depois que foi incubada”. E

“Alguns programas me chamaram a atenção (...)"

Na Tabela 1 está representado, de acordo com a tabulação do questionário aplicado via correio eletrônico, os suportes que, segundo os empresários incubados e graduados, foram mais atrativos para o ingresso no processo de incubação. Em seguida está reapresentada Tabela 2 que corresponde à escala somada para 12 respondentes e escala de 5 pontos.

Tabela 1: Apoios que estimularam o ingresso no processo de incubação

\begin{tabular}{|c|c|c|c|c|c|c|c|}
\hline Escala & \multicolumn{4}{|c|}{ Importante } & Guito & \multirow[t]{2}{*}{ Soma } & \\
\hline Apoios & 1 & 2 & 3 & 4 & 5 & & \\
\hline 1. Infraestrutura oferecida pela incubadora & 0 & 1 & 4 & 4 & 3 & 45 & \\
\hline 2. Acesso a infraestrutura da universidade & 2 & 3 & 1 & 3 & 3 & 38 & 2 \\
\hline 3. Capacitação do perfil empresarial & 1 & 2 & 4 & 3 & 2 & 39 & $\overline{0}$ \\
\hline 4. Consultorias oferecidas durante a incubação & 0 & 3 & 0 & 3 & 6 & 48 & ล. \\
\hline 5. Permissão para uso da marca CDT/UnB & 3 & 1 & 1 & 2 & 5 & 41 & \\
\hline 6. Acesso a outros programas do CDT/UnB & 3 & 3 & 2 & 4 & 1 & 36 & \\
\hline
\end{tabular}

Fonte: Elaboração própria 
Tabela 2: Escala somada para 12 respondentes e escala de 5 pontos

\begin{tabular}{|c|c|c|c|}
\hline Pouco Importante & & & Muito Importante \\
\hline $12-24$ & $25-36$ & $37-48$ & $49-60$ \\
\hline
\end{tabular}

Fonte: Elaboração própria

A Tabela 1 mostra que, de forma geral, os apoios oferecidos pela incubadora têm o mesmo grau de atração para os empresários incubados, pois a maioria deles se encontra no terceiro quartil (37-48), mais próximo do extremo positivo (muito importante). Salvador, Tutida e Ceretta (2009) encontraram em sua pesquisa que a infraestrutura oferecida é o apoio mais atrativo para os empresários. Esta pesquisa mostrou que para os empresários incubados no CDT/UnB a infraestrutura da incubadora, o acesso à universidade, a capacitação do perfil empresarial, as consultorias e as assessorias e a permissão do uso da marca CDT/UnB em materiais gráficos representam graus de atração próximos, sem destaque para nenhum deles, o que neste caso parece ser uma visão distinta àquele estudo.

O item "Acesso a outros programas do CDT/UnB” teve o menor score. Este apoio representa de forma característica a relação universidade-empresa, que é um dos fundamentos da hélice tríplice, e esta pesquisa constatou que na incubadora em estudo, o apoio que está diretamente ligado à relação universidade-empresa é também o menos atrativo para os empresários.

É preciso que haja um alinhamento entre o interesse da comunidade (empresário) e os programas oferecidos pela universidade. Como mostrou Zhou (2008) ao definir a relação empresa-universidade como tendo que estar baseada na necessidade e no consenso, ou seja, os serviços oferecidos pela universidade têm de estar alinhados às necessidades da indútria. Esta pesquisa constatou que os apoios da incubadora que estão mais intimamente relacionados com a universidade não são considerados relevantes para os empresários, o que vai de encontro à premissa proposta por Zhou(2008) e pode significar uma possível inadequação dos serviços oferecidos. Tal como indicado em uma das falas, há falta de conhecimento dos programas, o que pode ser uma falha da própria incubadora, em não estar divulgando junto às empresas sua capacidade plena de suporte. Ademais, esse resultado reforça a afirmação de Serra, Serra, Ferreira e Fiates (2010) que diz que as incubadoras devem ultrapassar a barreira dos serviços tradicionais e oferecerem serviços que gerem valor agregado. Dentre os serviços prestados pela incubadora em estudo percebeu-se que não há destaque para nenhum dos apoios oferecidos ou que para os empresários fosse especialmente atrativo. 


\subsection{Apoios que tiveram impacto na consolidação das empresas incubadas}

Este tópico trata dos suportes oferecidos pela incubadora que foram mais importantes para consolidação da empresa. Para ilustrar, serão transcritas algumas frases ditas pelos empresários incubados durante aplicação da metodologia The World Café e depois será apresentado o resultado do questionário aplicado aos empresários incubados e graduados.

Sobre os suportes oferecidos e a relação deles com o sucesso da empresa incubada, os empresários disseram que:

a) Infraestrutura oferecida pela incubadora:

“Ter uma sala dentro do CDT é muito bom, primeiro pelo preço que é baixo em relação aos imóveis na Asa Sul e Asa Norte e também porque a gente fica próximo ao pessoal da incubadora”.

b) Acesso à estrutura da universidade: laboratórios, professores, departamentos:

"Para quem precisa usar laboratórios... é muito bom, mas acho que a maioria das empresas não usou nenhum ainda (...)".

c) Capacitação do perfil empresarial,

"Alguns cursos são muito bons e tem efeito como é o caso do Empretec, mas outros, como o de relacionamento interpessoal, são pouco aprofundados."

d) Acesso a consultorias oferecidas durante o processo de incubação:

"As consultorias ajudam bastante, principalmente nas áreas de administração, mas a gente sente falta de consultorias na área da empresa".

e) Permissão para uso da marca CDT/UnB:

"Quando a gente coloca a marca num sítio eletrônico ou cartão dá certa credibilidade, mas não sei se é uma coisa que chega a ter muito impacto."

f) Acesso a outros programas do CDT/UnB:

"Quando a Empresa Y tentou contato com o NUPITEC pareceu que eles não queriam atender empresa incubada (...) teve muita burocracia”.

g) Parceria com outras empresas incubadas:

“A gente encontrou um parceiro aqui dentro da incubadora (...) a Empresa X trabalha com a Empresa Z há mais de um ano desenvolvendo o produto deles (...)”.

Tabela 3: Apoios que tiveram maior importância para a consolidação da empresa

\begin{tabular}{|c|ll|l|l|}
\hline Escala & Pouco & Muito & Soma & \\
\hline
\end{tabular}

Revista de Administração e Inovação, São Paulo, v. 10, n.3, p.208-229, jul./set. 2013. 


\begin{tabular}{|c|c|c|c|c|c|c|c|}
\hline \multirow[b]{2}{*}{ Apoios } & \multicolumn{5}{|c|}{ Importante Importante } & & \\
\hline & 1 & 2 & 3 & 4 & 5 & & \multirow{8}{*}{ 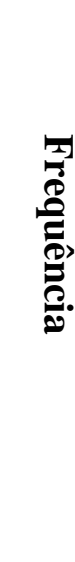 } \\
\hline 1. Infraestrutura oferecida pela incubadora & 0 & 4 & 3 & 2 & 3 & 40 & \\
\hline 2. Acesso a infraestrutura da universidade & 2 & 4 & 2 & 3 & 1 & 33 & \\
\hline 3. Capacitação do perfil empresarial & 0 & 5 & 2 & 4 & 1 & 37 & \\
\hline 4. Consultorias oferecidas durante a incubação & 1 & 2 & 2 & 5 & 2 & 41 & \\
\hline 5. Permissão para uso da marca CDT/UnB & 4 & 2 & 1 & 1 & 4 & 35 & \\
\hline 6. Acesso a outros programas do CDT/UnB & 6 & 3 & 0 & 2 & 1 & 25 & \\
\hline 7. Parceria com outras empresas incubadas & 1 & 3 & 1 & 3 & 4 & 42 & \\
\hline
\end{tabular}

Fonte: Elaboração própria.

Na Tabela 3 está representado, de acordo com a tabulação do questionário aplicado via $e$-mail, os suportes que, segundo os empresários incubados e graduados, tiveram maior importância para a consolidação das empresas incubadas. Ao se utilizar a Tabela 2 como parâmetro de interpretação da Tabela 3, evidencia-se que há diferenças entre os apoios relacionados à consolidação e aqueles que estimularam o ingresso. Chama a atenção que a infraestrutura da universidade, o uso da marca e o acesso a outros programas perderam importância na percepção dos empresários. Novamente, nenhum aspecto atingiu o quartil superior (muito importante) da escala somada. Parece que a necessidade de as incubadoras oferecerem serviços além dos tradicionais indicada no texto de Serra, Serra, Ferreira e Fiates (2010) é mais premente para a consolidação das empresas que para sua atração à incubação.

Para uma avaliação mais detalhada dos suportes que têm maior importância para a consolidação das empresas incubadas, foi feita outra análise das respostas dos empresários ao questionário, levando em consideração as empresas com maior e com menor faturamento. Foram consideradas as faixas "Até R\$ 5.000,00" e "Acima de R \$ 10.000,00", pois não houve nenhuma resposta para a faixa "Entre R \$ 5.000,00 e R\$ 10.000,00”. A Tabela5 mostra o resultado dessa segunda análise. Em seguida está representada Tabela 6 que corresponde à escala somada para nove respondentes e escala de cinco pontos. 
Tabela 4: Apoios que tiveram maior importância para a consolidação da empresa para empresas com maior faturamento

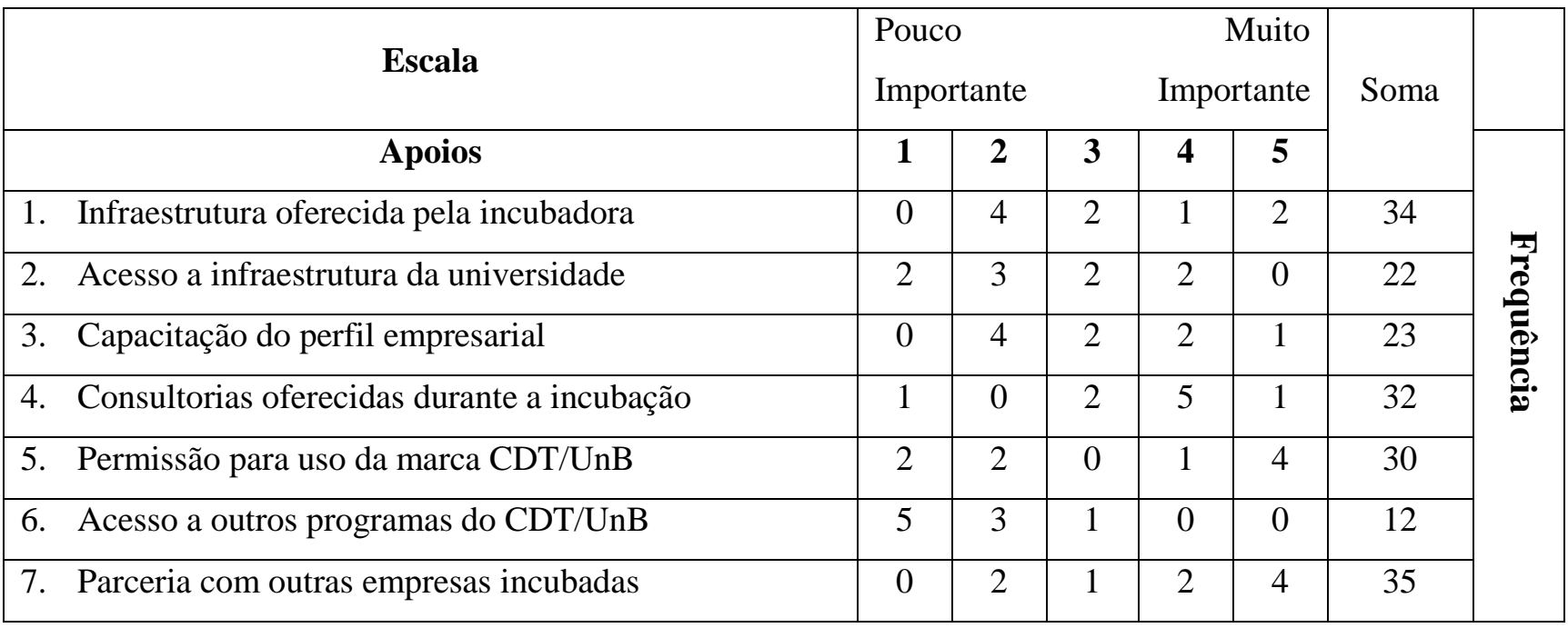

Fonte: Elaboração própria.

Tabela 5: Escala somada para 9 respondentes e escala de 5 pontos

\begin{tabular}{|c|c|c|c|}
\hline \multicolumn{4}{|l|}{ Pouco Importante } \\
\hline $09-18$ & $19-27$ & $28-36$ & $37-45$ \\
\hline
\end{tabular}

Fonte: Elaboração própria.

A comparação das duas análises mostrou diferenças entre as opiniões dos empresários. Na primeira análise, a infraestrutura da incubadora, capacitação do perfil empresarial, consultorias oferecidas e parceria com outras empresas incubadas ficaram na terceira faixa (37-48), próximas ao extremo positivo (Muito Importante). Enquanto o acesso à infraestrutura da universidade, uso da marca CDT/UnB e acesso a outros programas do CDT/UnB ficaram na segunda faixa (25-37), mais próximo ao extremo negativo (Pouco Importante).

Entretanto, quando foi levado em consideração o faturamento da empresa, houve mudança considerável no resultado. Segundo os representantes das empresas com maior faturamento, os apoios mais importantes para a consolidação da empresa foram a infraestrutura da incubadora, as consultorias oferecidas, uso da marca CDT/UnB e as parcerias com outras empresas incubadas, esses suportes ficaram na terceira faixa (28-36). O acesso à infraestrutura da universidade e a capacitação do perfil empresarial ficaram na segunda faixa (19-27) e o acesso a outros programas do CDT/UnB ficou na primeira faixa (09$18)$.

As semelhanças entre as duas análises foram que em ambas, a infraestrutura da incubadora, as consultorias e a parceria com outras empresas incubadas foram considerados os apoios mais importantes

Revista de Administração e Inovação, São Paulo, v. 10, n.3, p.208-229, jul./set. 2013. 
para a consolidação da empresa. Entretanto, em nenhuma das duas análises algum apoio se posicionou no quarto quartil.

Tabela 6: Apoios que tiveram maior importância para a consolidação da empresa para empresas com menor faturamento (até $\mathbf{R} 4 \mathbf{5 . 0 0 0 , 0 0 )}$

\begin{tabular}{|c|c|c|c|c|c|c|c|}
\hline Escala & \multicolumn{3}{|c|}{$\begin{array}{l}\text { Pouco } \\
\text { Importante }\end{array}$} & \multicolumn{2}{|c|}{$\begin{array}{r}\text { Muito } \\
\text { Importante }\end{array}$} & \multirow[t]{2}{*}{ Soma } & \multirow{9}{*}{ 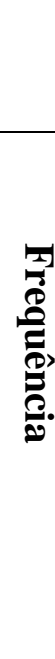 } \\
\hline Apoios & 1 & 2 & 3 & 4 & 5 & & \\
\hline 1. Infraestrutura oferecida pela incubadora & 0 & 0 & 1 & 1 & 1 & 12 & \\
\hline 2. Acesso a infraestrutura da universidade & 0 & 1 & 0 & 1 & 1 & 11 & \\
\hline 3. Capacitação do perfil empresarial & 0 & 1 & 0 & 2 & 0 & 10 & \\
\hline 4. Consultorias oferecidas durante a incubação & 0 & 2 & 0 & 0 & 1 & 9 & \\
\hline 5. Permissão para uso da marca CDT/UnB & 2 & 1 & 1 & 0 & 0 & 5 & \\
\hline 6. Acesso a outros programas do CDT/UnB & 1 & 0 & 1 & 0 & 1 & 9 & \\
\hline 7. Parceria com outras empresas incubadas & 1 & 0 & 0 & 1 & 0 & 7 & \\
\hline
\end{tabular}

Fonte: Elaboração própria.

Tabela 7: Escala somada para 9 respondentes e escala de 5 pontos

Pouco Importante

$$
03-06
$$

$$
01-09
$$

Muito Importante

$13-15$

\section{Fonte: Elaboração própria.}

Levando-se em consideração apenas as empresas com menor faturamento (Tabelas 6 e 7) os apoios "infraestrutura oferecida pela incubadora", "acesso a infraestrutura da universidade" e "capacitação do perfil empresarial" ficaram na terceira faixa (10-12) tendendo a "muito importante", os apoios "consultorias oferecidas", "acesso a outros programas do CDT/UnB" e "parceria com outras empresas incubadas" ficaram na segunda faixa (07-09) e o apoio menos relevante para a consolidação segundo empresas com menor faturamento foi “permissão para uso da marca CDT/UnB". Percebe-se que os apoios considerados mais relevantes estão ligados à infraestrutura e capacitação (terceiro quartil), ainda que também não haja registro de nenhum deles como muito importante (quarto quartil). Parece natural que as empresas com menor faturamento necessitem mais de infraestrutura e por isso recolheu-se essa percepção.

Comparando os resultados das duas análises (Quadro 3), pode-se perceber uma divergência com os resultados das empresas com maior faturamento. As empresas com maior faturamento parecem utilizar 
os apoios que são mais próprios de uma incubadora de base tecnológica. Ou seja, parece que estão mais próximas de se apropriar daqueles serviços que podem agregar valor ao seu produto tecnológico, como apontam Serra, Serra, Ferreira e Fiates (2010).

\begin{tabular}{|c|l|l|}
\hline Quartil & \multicolumn{1}{|c|}{ Empresas com maior faturamento } & \multicolumn{1}{|c|}{ Empresas com menor faturamento } \\
\hline $\mathbf{1}^{\mathbf{o}}$ & Acesso a outros programas do CDT/UnB & Uso da marca CDT/UnB \\
\hline \multirow{2}{*}{$\mathbf{2}^{\mathbf{o}}$} & $\begin{array}{l}\text { Acesso a infraestrutura da universidade. } \\
\text { Capacitação do perfil empresarial. }\end{array}$ & $\begin{array}{l}\text { Consultorias oferecidas. } \\
\text { Acesso a outros programas do CDT/UnB. } \\
\text { Parcerias com outras empresas incubadas. }\end{array}$ \\
\hline \multirow{\mathbf{3}^{\mathbf{o}}}{*}{} & $\begin{array}{l}\text { Infraestrutura da incubadora. } \\
\text { Consultorias oferecidas. }\end{array}$ & $\begin{array}{l}\text { Infraestrutura da incubadora. } \\
\text { Acesso a infraestrutura da universidade. } \\
\text { Capacitação do perfil empresarial. }\end{array}$ \\
\hline $\mathbf{4}^{\mathbf{0}}$ & $\begin{array}{l}\text { Parcerias com outras empresas incubadas. } \\
\mathbf{2}\end{array}$ & Sem ocorrência. \\
\hline
\end{tabular}

Quadro 3: Comparativo dos resultados de empresas com maior e menor faturamento.

Fonte: Elaboração própria.

O MCTI (2000) em seu Manual de Implantação de Incubadoras define que as incubadoras de empresas, entre outros, devem promover cultura empreendedora, a interação entre micro e pequenas empresas incubadas e estimular a associação entre pesquisadores e empresários. Silva (2009) descreve que para os empreendedores, as vantagens da incubação são advindas de questões como a aproximação do conhecimento teórico e a prática, estabelecimentos de redes estratégicas de relacionamento, incentivo às práticas gerenciais para a competitividade, acesso a equipamentos para pesquisa e desenvolvimento e redução de custos operacionais.

Esta pesquisa se assemelhou em alguns aspectos com o que escreveu Silva (2009), pois os empresários julgam como importante a infraestrutura da incubadora, as consultorias e a rede de relacionamento formada com outros empresários incubados, entretanto, os resultados desta pesquisa discordam de Silva (2009) quando mostram que para as empresas incubadas com maior faturamento, o acesso à universidade, por meio da qual poderiam utilizar laboratórios e equipamentos, e a capacitação empresarial são considerados pouco importantes para a consolidação da empresa.

Deve-se ter em conta que isso talvez se dê mesmo pela atividade fim da empresa. Na fala de um dos empresários foi citada a falta de consultoria na área de atuação da empresa. Lima \& Kuhl (2006) descreveu que entre os fatores que constituem obstáculos na implantação de produtos ou processos Revista de Administração e Inovação, São Paulo, v. 10, n.3, p.208-229, jul./set. 2013. 
inovadores estão a falta de serviços técnicos especializados, pouca informação sobre mercado e necessidades dos clientes e escassez de informações sobre tecnologia. Neste ponto, observou-se uma carência nos serviços prestados pela incubadora: falta de consultoria tecnológica para as empresas incubadas, o que parece evidenciar justamente os fatores que sustentam a incubação de empresa: seu monitoramento e avaliação como apontam Patton, Warren e Bream (2009).

\section{CONCLUSÕES E RECOMENDAÇÕES}

O objetivo geral desta pesquisa foi avaliar as formas de apoio como atrativo para o ingresso no processo de incubação e para a consolidação das empresas. De forma geral o que se pode perceber neste caso é que os empresários buscam, antes de estarem incubados, orientação posto que descrevem as consultorias como apoio mais atrativo, e o estabelecimento físico da empresa na incubadora. Ao passo que no segundo momento, já incubados, percebem que a rede de relacionamentos (parceria com outras empresas incubadas) é um dos fatores mais importante para a consolidação da empresa. Parece relevante destacar que as empresas com menor faturamento continuam necessitando muito mais de aspectos ligados à infraestrutura, talvez por seu próprio fluxo de caixa.

Mesmo assim, o que ocorre com as empresas de modo geral parece natural, na medida em que a necessidade de infraestrutura é premente para a criação de empresas e, uma vez satisfeita ela passa a ser secundária. O tema da rede de relacionamentos tem avançado no sentido de explicar que mesmo empresas fora da incubação estão buscando estabelecer algum tipo de rede, como indicou Franco (2007). Assim, não se pode considerar uma surpresa que ele tenha também aparecido como um apoio importante para a consolidação das empresas.

Uma conclusão que parece merecer destaque se relaciona ao suporte que a incubadora pode ter em termos de outros programas e mesmo o acesso à infraestrutura da Universidade. Parece que a incubadora estudada está subutilizada, principalmente ao se levar em conta a possibilidade de interação que pode surgir como o uso de outras infraestruturas, como os diversos laboratórios que a universidade possui. Isso pode ter sua origem na falta de experiência dos empresários na busca desse tipo de apoio, na falta de divulgação desse tipo de serviço, ou então ambas as coisas.

Por fim, os apoios atrativos e os que impactam sobre a consolidação da empresa ficaram claros nesta pesquisa, entretanto, os resultados refletem a realidade de uma incubadora de empresas. Mas esse 
estudo está limitado pelo número de respondentes em relação ao grupo alvo. Assim uma linha de pesquisa pode ser a complementação desses resultados na própria Incubadora em estudo.

Ainda, para ampliar a discussão sobre esses resultados e que reflitam uma realidade regional ou até nacional, é preciso que pesquisas como esta sejam realizadas em outras incubadoras. Isso possibilitaria tomadas de decisão mais adequadas por parte da gerência das incubadoras, por um lado, e a compreensão melhor daqueles aspectos que pesam mais à hora de atrair e ou consolidar uma empresa junto a uma incubadora.

\section{REFERÊNCIAS}

Abdalla, M. M., Calvosa, M. V. D., \& Batista, L. V. (2009). Hélice Tríplice no Brasil: Um ensaio teórico acerca dos benefícios da entrada da universidade nas parcerias estatais. Revista Cadernos da Administração da Faculdade Salesiana Maria Auxiliadora. 1, 34-52.

Andino, B. F. A., Fracasso, E.M., Silva, P.G.L. \& Lobler, M.L. (2004). Avaliação do processo de incubação de empresas em incubadoras de base tecnológica. Anais do Enampad, Brasil, 28, s.p.

Associação Nacional de Entidades Promotoras de Empreendimentos Inovadores - Anprotec (2002 setembro). Glossário dinâmico de termos na área de tecnopolos, parques tecnológicos e incubadoras de empresas. Recuperado em 13 novembro, 2010 do sítio web Anprotec: www.anprotec.org.br.

Associação Nacional de Entidades Promotoras de Empreendimentos Inovadores - Anprotec (2007). Aventura do possível: passado, presente e futuro de um movimento que há 20 anos acredita em Inovação $e$ Empreendedorismo no Brasil. Recuperado em 10 novembro, 2010 do sítio web Anprotec: www.anprotec.org.br.

Associação Nacional de Entidades Promotoras de Empreendimentos Inovadores - Anprotec (2011). $O$ que é uma incubadora? Recuperado em 25 setembro, 2013 do sítio web Anprotec: http://anprotec.org.br/site/pt/incubadoras-e-parques/perguntas-frequentes/.

Brown,J., Isaacs, D. O. (2007). The world café: dando forma ao nosso futuro por meio de conversações significativas e estratégicas. São Paulo: Cultrix.

Bulla, F. J. M. \& Gartner, C. A. C. (2004). La experiencia colombiana en incubación de empresas. Revista-Escuela de Administración de Empresas, 502, 7-13.

Centro de Apoio ao Desenvolvimento Tecnológico - CDT. Sítio web, acesso em dezembro, 10, 2010, em www.cdt.unb.br.

Centro de Apoio ao Desenvolvimento Tecnológico - CDT. (2010)Edital de Seleção 2010. Diário Oficial da União, 277, Seção 3, 38. Recuperado em 12 de novembro de 2010 de http://portal.in.gov.br/ 
Chandra, A. B. \& Fealey, T. (2009, December). Business incubation in United States, China and Brazil: a comparison of role of government, incubation funding and financial services. International Journal of Entrepreneurship. 13, s.p.

Dagnino, R. (2003). A relação universidade-empresa no Brasil e o "Argumento da Hélice Tríplice". Revista Brasileira de Inovação. 2,267-307.

Dornelas, J. C. (2002). Planejando incubadoras de empresas. Rio de Janeiro: Editora Campus.

Franco, M. J. B. (2007). Tipologia de processos de cooperação empresarial: uma investigação empírica sobre o caso português, Revista de Administração Contemporânea, 11, 149-176.

Hackett, S.M. \& Dilts, D. M. (2004). A systematic review of business incubation research. Journal on Technology Transfer, Netherland. 29, 55-84.

Hernández, P. P. \& Estrada, A. M. (2006). Análisis del sistema de incubación de empresas de base tecnológica de México. Anais do Congreso Iberoamericana de Ciencia, Tecnología e Innovación CTS+I, 1. México, s.p. .

Instituto Brasileiro de Geografia Estatística- IBGE \& (2008). PINTEC - Pesquisa de inovação tecnológica: 2008. Rio de Janeiro: IBGE, 2010.

Lima, L.F. \& Kuhl, M.R. (2006). Obstáculos na implementação de inovações. Anais do Simpósio da Gestão da Inovação Tecnológica, 26. Brasil, s.p..

Lu, L. (2008). Creating knowledge-base innovation China. Journal of Technology Management. 3, 249263.

Ministério da Ciência, Tecnologia e Inovação - MCTI (novembro, 2000). Manual para implantação de incubadoras de empresas. Recuperado em 10 novembro, 2010 do sítio web: www.mct.gov.br.

Medeiros, J.A. (1998). Incubadoras de empresas: lições da experiência internacional. Revista de Administração, 33, 5-20.

Moraes, C. S. \& Firmo, D. O. (2004). O papel das incubadoras de empresas no estabelecimento de arranjos produtivos estruturados. Anais do Simpósio da Gestão Tecnológica, 23. Brasil, s.p.National Business Incubation Association - NBIA (2009). The history of business incubation. Recuperado em 7 janeiro, 2009 de www.nbia.org.

Oliva, F.L., Sobral, M.C.; Santos, S.A.; Almeida, M.I.R. \& Grisi, C.C.H. (2011). Measuring the probability of innovation in technology-based companies. Journal of Manufacturing Technology Management..22, 3, 365-383.

Patton, D., Warren, L. \& Bream, D. (2009). Elements that underpin hight-tech business incubation process. Journal of Technology Transfer, 34, 621-636.

Raupp, F.M. \& Beuren, I.M. (2006). O suporte das incubadoras brasileiras para potencializar as características empreendedoras nas empresas incubadas. Revista de Administração,.41, 419-430.

Revista de Administração e Inovação, São Paulo, v. 10, n.3, p.208-229, jul./set. 2013. 
Roesch, S. M. A. (2006). Projetos de estágio e de pesquisa em Administração.. São Paulo: Editora Atlas.

Salvador, A.L., Tutida, L. \& Ceretta, G.F. (2009). Incentivos concedidos por incubadoras e suas influências no desempenho de MPES de base tecnológica: O caso do hotel tecnológico da UTFPR de Cornélio Procópio - PR. Diálogos \& Saberes.. 5, pp. 31-46.

Serra, B. C., Serra, F. A. R; Ferreira, M. PP. \& Fiates, G. G. (2010, abril) Factores fundamentais para o desempenho das incubadoras. Revista de Administração e Inovação, 8, 221-247.

Sistema Brasileiro de Apoio a Micro e Pequenas Empresas - SEBRAE (2004). Fatores Condicionantes $e$ Taxa de Mortalidade de Empresas no Brasil. Recuperado em 13 novembro, 2010 do sítio web www.sebrae.org.br.

Silva, S. A. (2009). A relevância das incubadoras no mundo contemporâneo. Ponto e Vírgula, 6, 235-251.

Studdard, N.L. (2006). The effectiveness of entrepreneurial firm's knowledge acquisition from a business incubator. International Entrepreneuship Management Journal, United States. 2, 211-225.

Tang, M., Angathevar, B. \& Pancholi, J. (2010). Technology business incubation in China and India: a comparative analysis. Annals of International Conference Making Innovation. Work for Society: Linking, Leveraging and Learning, 8, Malásia.

Trevisan, M. \& Silva, T.N. (2010). Programa primeira empresa inovadora: Uma possibilidade de operacionalização da hélice tríplice no Brasil. Anais do Simpósio da Gestão da Inovação Tecnológica, 26, Brasil..

Vedovello, C. \& Figueiredo, P.N. (2005). Incubadora de inovação: que nova espécie é essa? Revista de Administração de Empresas (RAE eletrônica), 4, s.p.

Vergara, S.M. (2006). Projetos e relatórios de pesquisa em administração. São Paulo: Atlas.

Wolffenbüttel, A. P. (2001). Avaliação do processo de interação universidade-empresa em incubadoras universitárias de empresas: um estudo de caso na incubadora de empresas de base tecnológica da UNISINOS. Dissertação de mestrado não publicada Universidade Federal do Rio Grande do Sul, Porto Alegre.

Zhou, C. (2008). Emergence of the entrepreneurial university in evolution of the triple helix: The case of Northeastern University in China. Journal of Technology Management in China, 3, 109-126.

Pequenos investimentos em novas iniciativas empresariais (FNABA).

Data do recebimento do artigo: 29/04/2013

Data do aceite de publicação: 07/09/2013

Revista de Administração e Inovação, São Paulo, v. 10, n.3, p.208-229, jul./set. 2013. 